\title{
EVOLUTIONARY SCENARIOS FOR DOUBLE DEGENERATE SYSTEMS
}

\author{
P. B. MARKS ${ }^{1}$, M. J. SARNA ${ }^{1,2}$, R. C. SMITH ${ }^{1}$ \\ 1. Astronomy Centre, University of Sussex, Brighton, UK \\ 2. N. Copernicus Astronomical Center, Warsaw, Poland
}

\section{Observations}

There are presently eight double degenerate systems with well determined orbital parameters, their periods being either a few hours or a few days (Marsh, Dhillon \& Duck 1995; Marsh 1995). The masses of the primaries and secondaries lie in the range $0.15 \ldots 0.45 \mathrm{M}_{\odot}$.

\section{Calculations}

We calculate two evolutionary scenarios (Sarna, Marks \& Smith 1996); the first is Algol-type evolution with two phases of stable mass transfer, and the second involves first a stage of common envelope $(\mathrm{CE})$ evolution followed by a stage of stable mass transfer. In both calculations we assume non-conservative mass transfer by which we mean that the total mass and angular momentum of the system are not conserved. For both scenarios we start our calculations after the first stage of mass transfer has finished. In all calculations the primary is the initially more massive star that filled its Roche lobe and transferred material to the secondary during the first phase of mass transfer, hence the secondary is the star that fills its Roche lobe in our calculations. The system's orbital period decreases and then increases until the system detaches; we are left with a detached white dwarf/white dwarf binary with an orbital period of the order of hours or of days (see Table 1). There must exist some bifurcation period below which the systems evolve towards orbital periods of the order of hours and above which the systems evolve to periods of the order of several days. 
TABLE 1. Results of model computations

\begin{tabular}{lccccc} 
Model No. & 1 & 2 & 3 & 4 & Algol \\
\hline $\mathrm{P}_{\mathrm{i}}[\mathrm{d}]$ & 0.950 & 1.000 & 1.263 & 1.612 & 2.664 \\
$\mathrm{M}_{1 \mathrm{i}} / \mathrm{M}_{\odot}$ & 0.250 & 0.250 & 0.250 & 0.250 & 0.207 \\
$\mathrm{M}_{2 \mathrm{i}} / \mathrm{M}_{\odot}$ & 1.750 & 1.750 & 1.750 & 1.750 & 1.920 \\
$\mathrm{M}_{\mathrm{He} 2 \mathrm{i}} / \mathrm{M}_{\odot}$ & 0.000 & 0.000 & 0.032 & 0.072 & 0.098 \\
$\mathrm{P}_{\mathrm{f}}[\mathrm{d}]$ & 0.176 & 0.395 & 1.620 & 2.556 & 2.636 \\
$\mathrm{M}_{1 \mathrm{f}} / \mathrm{M}_{\odot}$ & 0.411 & 0.409 & 0.404 & 0.405 & 0.409 \\
$\mathrm{M}_{2 \mathrm{f}} / \mathrm{M}_{\odot}$ & 0.145 & 0.158 & 0.206 & 0.198 & 0.286 \\
$\mathrm{M}_{\mathrm{He} 2 \mathrm{f}} / \mathrm{M}_{\odot}$ & 0.068 & 0.086 & 0.097 & 0.147 & 0.125 \\
$\log$ Time $[\mathrm{yr}]$ & 8.692 & 8.607 & 7.900 & 7.906 & 6.901 \\
\hline
\end{tabular}

Subscript $i$ denotes the initial parameters, subscript $f$ denotes detachment and subscript He2 denotes the secondary's helium core. The duration of the semi-detached evolution is given by log Time.

\section{Conclusions}

We can explain the separation/segregation of the orbital periods of double degenerate systems using the bifurcation 'mechanism'. This 'mechanism' also works in a similar way for low-mass binaries with millisecond pulsars and white dwarf components (Ergma \& Sarna 1996). We can see that our theoretical models reproduce the general features of the observational data very well, but more accurate orbital parameters will be necessary before we can model the evolutionary history of particular systems.

\section{References}

Ergma, E., Sarna, M.J., 1996, MNRAS, in press

Marsh, T.R., Dhillon, V.S., Duck, S.R, 1995, MNRAS, 275, 828

Marsh, T.R., 1995, MNRAS, 275, L1

Sarna, M.J., Marks, P.B., Smith, R.C., 1996, MNRAS, in press 